\title{
Direct detection of dark matter in the minimal supersymmetric standard model with non-universal Higgs boson masses
}

\author{
John Ellis, ${ }^{1}$ Andy Ferstl, ${ }^{2}$ Keith A. Olive,${ }^{3}$ and Yudi Santoso ${ }^{3}$ \\ ${ }^{1}$ TH Division, CERN, Geneva, Switzerland \\ ${ }^{2}$ Department of Physics, Winona State University, Winona, Minnesota 55987 \\ ${ }^{3}$ William I. Fine Theoretical Physics Institute, University of Minnesota, Minneapolis, Minnesota 55455
}

(Received 7 February 2003; published 2 June 2003)

\begin{abstract}
We calculate dark matter scattering rates in the minimal supersymmetric extension of the standard model (MSSM), allowing the soft supersymmetry-breaking masses of the Higgs multiplets, $m_{1,2}$, to be nonuniversal (NUHM). Compared with the constrained MSSM (CMSSM) in which $m_{1,2}$ are required to be equal to the soft supersymmetry-breaking masses $m_{0}$ of the squark and slepton masses, we find that the elastic scattering cross sections may be up to two orders of magnitude larger than values in the CMSSM for similar lightest supersymmetric particle masses. We find the following preferred ranges for the spin-independent cross section, $10^{-6} \mathrm{pb} \gtrsim \sigma_{S I} \gtrsim 10^{-10} \mathrm{pb}$, and, for the spin-dependent cross section, $10^{-3} \mathrm{pb} \gtrsim \sigma_{S D}$, with the lower bound on $\sigma_{S I}$ dependent on using the putative constraint from the muon anomalous magnetic moment. We stress the importance of incorporating accelerator and dark matter constraints in restricting the NUHM parameter space, and also of requiring that no undesirable vacuum appear below the grand unified theory (GUT) scale. In particular, values of the spin-independent cross section another order of magnitude larger would appear to be allowed, for small $\tan \beta$, if the GUT vacuum stability requirement were relaxed, and much lower cross-section values would be permitted if the muon anomalous magnetic moment constraint were dropped.
\end{abstract}

DOI: 10.1103/PhysRevD.67.123502

PACS number(s): 95.35.+d, 12.60.Jv, 14.80.Ly

\section{INTRODUCTION}

There have been many previous studies of the elastic scattering rates of supersymmetric relic particles on matter in the minimal supersymmetric extension of the standard model (MSSM) [1-8], assuming conservation of $R \equiv(-1)^{3 B+L+2 S}$, where $B$ is the baryon number, $L$ the lepton number and $S$ the spin, so that the lightest supersymmetric particle (LSP) is absolutely stable. As in most previous studies, we assume this to be the lightest neutralino $\chi$ [9]. In this paper, we refine and extend previous calculations (see, for example, $[2,4,8]$ ) of the elastic scattering rates when the input soft supersymmetry-breaking scalar masses for the Higgs multiplets are allowed to be nonuniversal at the input grand unified theory (GUT) scale, the nonuniversal Higgs model (NUHM).

As we discuss later in more detail, it is important to impose the constraints due to accelerator experiments, including searches at the CERN $e^{+} e^{-}$collider LEP, $b \rightarrow s \gamma$ and (optionally) the muon anomalous magnetic moment, $g_{\mu}$ -2 . We assume also that most of the cold dark matter is composed of LSPs, with relic density $0.1<\Omega_{\chi} h^{2}<0.3$, while being aware that the lower part of this range currently appears the most plausible [10]. In particular, including the recent Wilkinson Microwave Anistropy Probe (WMAP) data [11] (which appeared after submission of this paper), the range $0.094<\Omega_{\chi}<0.129$ is preferred (though see [12] for a skeptical discussion) and we comment later on the implications of restricting attention to this narrower band of $\Omega_{\chi} h^{2}$.

In the constrained MSSM (CMSSM), in which all the soft supersymmetry-breaking scalar masses $m_{0}$ are assumed to be universal, including those for the Higgs doublets $H_{1,2}$, the underlying parameters may be taken as $m_{0}$, the soft supersymmetry-breaking gaugino mass $m_{1 / 2}$ which is assumed to be universal, the trilinear supersymmetry-breaking parameters $A_{0}$ that we set to zero at the GUT scale in this paper, and the ratio $\tan \beta$ of Higgs vacuum expectation values. In the NUHM [13-17], there are two additional free parameters, the two soft Higgs boson masses or equivalently the Higgs superpotential coupling $\mu$ and the pseudoscalar Higgs boson mass $m_{A}$. These would be fixed by the electroweak symmetry-breaking vacuum conditions in the CMSSM, up to a sign ambiguity in $\mu$, in terms of the other parameters $\left(m_{0}, m_{1 / 2}, A_{0}, \tan \beta\right)$. We use the parameters $\left(m_{0}, m_{1 / 2}, \mu, m_{A}, A_{0}, \tan \beta\right)$ to parametrize the more general NUHM.

As we have pointed out previously [16,17], this sixdimensional NUHM parameter space is significantly restricted by the requirement that no undesirable vacuum appears when one uses the renormalization-group equations to run the soft supersymmetry-breaking parameters between the input GUT scale and the electroweak scale. This requirement constrains the nonuniversalities of the Higgs boson masses: $\hat{m}_{i} \equiv \operatorname{sgn}\left(m_{i}^{2}\right)\left|m_{i} / m_{0}\right|: i=1,2$, which in turn restricts the range of elastic scattering cross sections that we find. ${ }^{1}$

The allowed regions in the $\left(\mu, m_{A}\right),\left(\mu, M_{2}\right)$ and $\left(m_{1 / 2}, m_{0}\right)$ planes for certain discrete values of the other NUHM parameters have been described in [17]. Our first step in this paper is to provide contours of the elastic scattering cross sections in selected planes, providing a first

\footnotetext{
${ }^{1}$ This extended stability requirement would also exclude nonuniversalities for the input squark and slepton masses that allowed their physical values to be similar, an assumption that might lead to much larger elastic scattering cross sections than we find here.
} 
comparison with the CMSSM points that appear in these planes. Secondly, we display the ranges of elastic scattering cross sections that are allowed in these planes, as functions of the LSP mass. In general, we find that the spinindependent elastic scattering cross sections may be up to two orders of magnitude larger than values in the CMSSM for similar LSP masses, and another order of magnitude larger if the GUT vacuum stability requirement is relaxed. Thirdly, we display ranges of the elastic scattering cross sections as functions of the LSP mass for all allowed values of the other NUHM parameters. Our improvements over previous work include an up to date implementation of the phenomenological constraints as well as a more conservative approach to the GUT stability constraint, as described in more detail below.

We review the NUHM in Sec. II, including the experimental and phenomenological constraints on its parameter space. Then, in Sec. III, we summarize our treatment of the elastic scattering matrix elements and display contours of the cross sections in various planar projections of the NUHM parameter space. Section IV presents and discusses the ranges of the cross sections attainable in the NUHM. Finally, Sec. V draws some conclusions from our analysis.

\section{THE NUHM AND CONSTRAINTS ON ITS PARAMETER SPACE}

We assume that the soft supersymmetry-breaking parameters are specified at some large input scale $M_{X}$, such as the supergravity or grand unification scale. Motivated by restrictions on flavor-changing neutral interactions, we assume that squarks and sleptons with the same standard model quantum numbers have universal soft supersymmetry-breaking scalar masses at this input scale. With the weaker justification provided by some GUTs, we further assume universality between the soft scalar masses of squarks and sleptons. However, in the NUHM studied here, we allow the soft supersymmetry-breaking scalar contributions to the masses of the Higgs supermultiplets at $M_{X}$ to be free nonuniversal parameters. Their running from $M_{X}$ down to low energies relates $m_{1}^{2}\left(M_{X}\right)$ and $m_{2}^{2}\left(M_{X}\right)$ to the Higgs boson supermultiplet mixing parameter $\mu$ and the pseudoscalar Higgs boson mass $m_{A}$. Therefore, we use as free parameters $\mu\left(m_{Z}\right) \equiv \mu$ and $m_{A}(Q) \equiv m_{A}$, where $Q \equiv\left(m_{\tilde{t}_{R}} m_{\tilde{t}_{L}}\right)^{1 / 2}$, in addition to the parameters $\left(m_{0}\left(M_{X}\right), m_{1 / 2}\left(M_{X}\right), A_{0}, \tan \beta\right)$ used in the CMSSM. $^{2}$

We note that, in several previous studies $[8,14]$, nonuniversalities have been parametrized by deviation parameters, $\delta_{i}$, defined by $m_{i}^{2} \equiv\left(1+\delta_{i}\right) m_{0}^{2}$. In a previous paper [16], the relation between the mass ratios, $\left(1+\delta_{i}\right)$, and $\mu$ and $m_{A}$ were given when other supersymmetric parameters are fixed. Here we have chosen the more physical low energy parameters as inputs rather than the $\delta_{i}$.

\footnotetext{
${ }^{2}$ In this paper, we use the input $A_{0}=0$ for definiteness, noting that the range of effective low-energy values of $A$, after renormalization below the GUT scale, is quite limited.
} form:

The electroweak vacuum conditions may be written in the

$$
m_{A}^{2}(Q)=m_{1}^{2}(Q)+m_{2}^{2}(Q)+2 \mu^{2}(Q)+\Delta_{A}(Q)
$$

and

$$
\mu^{2}=\frac{m_{1}^{2}-m_{2}^{2} \tan ^{2} \beta+\frac{1}{2} m_{Z}^{2}\left(1-\tan ^{2} \beta\right)+\Delta_{\mu}^{(1)}}{\tan ^{2} \beta-1+\Delta_{\mu}^{(2)}},
$$

where $\Delta_{A}$ and $\Delta_{\mu}^{(1,2)}$ are loop corrections [18-21] and $m_{1,2}$ $\equiv m_{1,2}\left(m_{Z}\right)$. We incorporate the known radiative corrections $[19,22,23] c_{1}, c_{2}$ and $c_{\mu}$ relating the values of the NUHM parameters at $Q$ to their values at $m_{Z}$ :

$$
\begin{aligned}
& m_{1}^{2}(Q)=m_{1}^{2}+c_{1} \\
& m_{2}^{2}(Q)=m_{2}^{2}+c_{2} \\
& \mu^{2}(Q)=\mu^{2}+c_{\mu} .
\end{aligned}
$$

Solving for $m_{1}^{2}$ and $m_{2}^{2}$, one has

$$
\begin{aligned}
m_{1}^{2}\left(1+\tan ^{2} \beta\right)= & m_{A}^{2}(Q) \tan ^{2} \beta-\mu^{2}\left(\tan ^{2} \beta+1-\Delta_{\mu}^{(2)}\right) \\
& -\left(c_{1}+c_{2}+2 c_{\mu}\right) \tan ^{2} \beta-\Delta_{A}(Q) \tan ^{2} \beta \\
& -\frac{1}{2} m_{Z}^{2}\left(1-\tan ^{2} \beta\right)-\Delta_{\mu}^{(1)}
\end{aligned}
$$

and

$$
\begin{aligned}
m_{2}^{2}\left(1+\tan ^{2} \beta\right)= & m_{A}^{2}(Q)-\mu^{2}\left(\tan ^{2} \beta+1+\Delta_{\mu}^{(2)}\right) \\
& -\left(c_{1}+c_{2}+2 c_{\mu}\right)-\Delta_{A}(Q)+\frac{1}{2} m_{Z}^{2}(1 \\
& \left.-\tan ^{2} \beta\right)+\Delta_{\mu}^{(1)}
\end{aligned}
$$

which we use to perform our numerical calculations. These quanties are necessary since here we are specifying boundary conditions for $\mu$ and $m_{A}$ at the weak scale. From these relations, the values of $m_{i}$ are run back up to the GUT scale where the remaining universal masses are reset. The process continues iteratively until convergence.

It can be seen from Eqs. (4) and (5) that, if $m_{A}$ is too small or $\mu$ is too large, then $m_{1}^{2}$ and/or $m_{2}^{2}$ can become negative and large. This could lead to $m_{1}^{2}\left(M_{X}\right)+\mu^{2}\left(M_{X}\right)$ $<0$ and/or $m_{2}^{2}\left(M_{X}\right)+\mu^{2}\left(M_{X}\right)<0$, thus triggering electroweak symmetry breaking at the GUT scale. The requirement that electroweak symmetry breaking occurs far below the GUT scale forces us to impose the conditions $m_{1}^{2}\left(M_{X}\right)$ $+\mu^{2}\left(M_{X}\right), m_{2}^{2}\left(M_{X}\right)+\mu^{2}\left(M_{X}\right)>0$ as extra constraints, which we call the GUT stability constraint. ${ }^{3}$ Note that this constraint is not equivalent to the constraint that $\delta_{i}>-1$ as is frequently imposed by others $[8,14]$. The $\delta_{i}$ here can be

\footnotetext{
${ }^{3}$ For a different point of view, however, see [24].
} 
large and negative so long as the above conditions are satisfied. In this sense our constraint represents a justifiable relaxation of the constraint and thus an enlargement of the allowable parameter space. We have discussed in [17] issues related to the NUHM renormalization group equations (RGE's) and their solutions.

We assume that $R$ parity is conserved, so that the LSP is stable and is present in the Universe today as a relic from the Big Bang. Searches for anomalous heavy isotopes tell us that the dark matter should be weakly interacting and neutral, and therefore eliminate all but the neutralino and the sneutrinos as possible LSPs. LEP and direct dark-matter searches together exclude a sneutrino LSP [25], at least if the majority of the CDM is the LSP. Thus we require in our analysis that the lightest neutralino be the LSP. We include in our analysis all relevant coannihilation processes involving this LSP and sparticles that may become near-degenerate in various regions of the NUHM parameter space. We restrict our attention to regions of the NUHM parameter space where 0.1 $<\Omega_{\chi} h^{2}<0.3$.

We impose in our analysis the constraints provided by direct sparticle searches at LEP, including that on the lightest chargino $\chi^{ \pm}: m_{\chi^{ \pm}} \gtrsim 103.5 \mathrm{GeV}$ [26], and that on the selectron $\tilde{e}: m_{\tilde{e}} \geq 99 \mathrm{GeV}$ [27]. Another important constraint is provided by the LEP lower limit on the Higgs boson mass: $m_{H}>114.4 \mathrm{GeV}[28]$ in the standard model. ${ }^{4}$ The lightest Higgs boson $h$ in the general MSSM must obey a similar limit, which may in principle be relaxed for larger $\tan \beta$. However, as we discussed in our previous analysis of the NUHM [16], the relaxation in the LEP limit is not relevant in the regions of MSSM parameter space of interest to us. We recall that $m_{h}$ is sensitive to sparticle masses, particularly $m_{\tilde{t}}$, via loop corrections $[29,30]$, implying that the LEP Higgs limit constrains the NUHM parameters. We also impose the constraint imposed by measurements of $b \rightarrow s \gamma$ [31], as discussed in [17].

We take an agnostic attitude towards the latest value of the anomalous magnetic moment of the muon reported [32] by the BNL E821 experiment. The world average of $a_{\mu}$ $\equiv \frac{1}{2}\left(g_{\mu}-2\right)$ now deviates by $(33.7 \pm 11.2) \times 10^{-10}$ from the standard model calculation of [33] using $e^{+} e^{-}$data, and by $(9.4 \pm 10.5) \times 10^{-10}$ from the standard model calculation of [33] based on $\tau$ decay data. On some of the subsequent plots, we display the formal $2-\sigma$ range $11.3 \times 10^{-10}<\delta a_{\mu}$ $<56.1 \times 10^{-10}$. However, in view of the checkered history of the theoretical standard model calculations of $a_{\mu}$, we do not impose this as an absolute constraint on the supersymmetric parameter space.

The results of applying the above constraints to various two-dimensional projections of the NUHM parameter space were described in [17].

\section{CONTOURS OF THE CROSS SECTIONS FOR ELASTIC SCATTERING}

The code we use to calculate the spin-independent and -dependent elastic dark matter scattering cross sections

\footnotetext{
${ }^{4}$ In view of the theoretical uncertainty in calculating $m_{h}$, we apply this bound with just three significant digits, i.e., our figures use the constraint $m_{h}>114 \mathrm{GeV}$.
}

$\sigma_{S I, S D}$ was documented in $[3,4]$, together with the ranges of values of the hadronic matrix elements that we use. The cross sections for protons and neutrons are similar within the quoted uncertainties in these matrix elements. There are other codes available [34] that include additional contributions to the scattering matrix elements, but a comparison [5] showed that the improvements were not essential for the CMSSM, and we believe they may also be neglected for our comparisons of the NUHM.

In [17], we analyzed NUHM dark matter in two ways: (i) fixing $\tan \beta=10$ and $\mu>0$, but choosing different values of $\mu$ and $m_{A}$, rather than assuming the CMSSM values, and (ii) varying $\tan \beta$ for representative fixed values of $\mu$ and $m_{A}$. We presented in [17] three types of slices through the NUHM parameter space, along $\left(m_{1 / 2}, m_{0}\right)$ planes, $\left(\mu, m_{A}\right)$ planes and $\left(\mu, M_{2}\right)$ planes. The latter is chosen instead of the $\left(\mu, m_{1 / 2}\right)$ plane, since there historically low energy supersymmetry has often been projected onto this plane.

In this paper, we concentrate first on a few specific examples of these slices, presenting later more general results. We choose two representative examples each of the $\left(m_{1 / 2}, m_{0}\right)$ planes, $\left(\mu, m_{A}\right)$ planes and $\left(\mu, M_{2}\right)$ planes shown previously. As we discuss later in more detail, the dependences of the cross sections on $\tan \beta$ are weaker than those on some other parameters, so we concentrate on planes for $\tan \beta=10$. The examples we choose are the $\left(m_{1 / 2}, m_{0}\right)$ planes for $\mu=400 \mathrm{GeV}, m_{A}=400 \mathrm{GeV}$ and $\mu$ $=700 \mathrm{GeV}, m_{A}=700 \mathrm{GeV}$, corresponding to Figs. 2(a) and (d) of [17], the $\left(\mu, m_{A}\right)$ planes for $m_{0}=100 \mathrm{GeV}, m_{1 / 2}$ $=300 \mathrm{GeV}$ and $m_{0}=300 \mathrm{GeV}, m_{1 / 2}=300 \mathrm{GeV}$, corresponding to Figs. 4(a) and (c) of [17], and the $\left(\mu, M_{2}\right)$ planes for $m_{0}=100 \mathrm{GeV}, m_{A}=300 \mathrm{GeV}$ and $m_{0}=300 \mathrm{GeV}, m_{A}$ $=500 \mathrm{GeV}$, corresponding to Figs. 8(a) and (c) of [17].

\section{A. Examples of $\left(m_{1 / 2}, m_{0}\right)$ planes}

We display in Fig. 1 contours of $(a, b)$ the spinindependent and $(\mathrm{c}, \mathrm{d})$ the spin-dependent elastic scattering cross sections, in the cases $\tan \beta=10$, and $(\mathrm{a}, \mathrm{c}) \mu$ $=400 \mathrm{GeV}$ and $m_{A}=400 \mathrm{GeV}$, and (b,d) $\mu=700 \mathrm{GeV}$ and $m_{A}=700 \mathrm{GeV}$. We assume here and in the subsequent figures that $A_{0}=0, m_{t}=175 \mathrm{GeV}$ and $m_{b}\left(m_{b}\right)_{S M}^{\overline{M S}}=4.25 \mathrm{GeV}$. Here and elsewhere, the thickest contours denote decades in the cross-section values in $\mathrm{pb}$, labeled by their exponents. The medium and thinnest lines are intermediate contours in the cross-section values, namely $2 \times$ and $5 \times$ decades, as labeled.

We notice immediately that the cross-section contours are nearly vertical at large $m_{1 / 2}$, reflecting the fact that they become almost independent of $m_{0}$ in the NUHM. We also notice that, within the GUT stability range (inside the black dot-dashed curves), the cross sections increase with $m_{1 / 2}$. This is because the LSP becomes more Higgsino-like as $m_{1 / 2}$ increases. However, the cross sections do decrease again for very large $m_{1 / 2}$ beyond the GUT stability limit as the low energy scalar masses increase with $m_{1 / 2}$ as does the light Higgs boson mass (though slowly).

The GUT stability requirement imposes $m_{1 / 2}$ $\$ 600(1100) \mathrm{GeV}$ in panels $(\mathrm{a}, \mathrm{c})$ and $(\mathrm{b}, \mathrm{d})$, respectively. Be- 

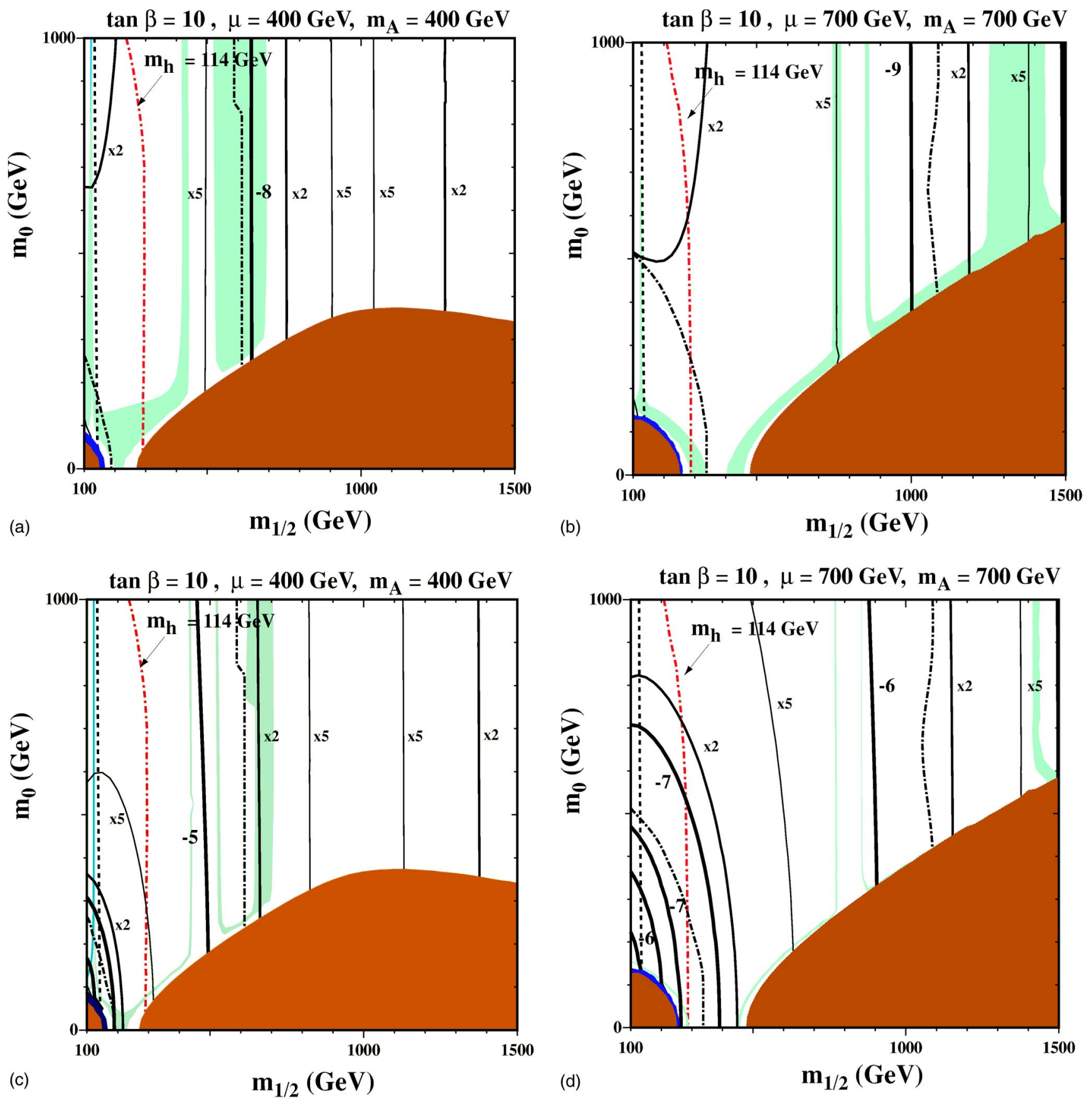

FIG. 1. Contours of $(\mathrm{a}, \mathrm{b})$ the spin-independent and $(\mathrm{c}, \mathrm{d})$ the spin-dependent cross sections (solid black lines) are superimposed on the NUHM $\left(m_{1 / 2}, m_{0}\right)$ planes for $\tan \beta=10$ and $(\mathrm{a}, \mathrm{c}) \mu=400 \mathrm{GeV}$ and $m_{A}=400 \mathrm{GeV}$, and (b,d) $\mu=700 \mathrm{GeV}$ and $m_{A}=700 \mathrm{GeV}$. The near-vertical dot-dashed lines are the contours $m_{h}=114 \mathrm{GeV}$, and the near-vertical dashed lines at lower $m_{1 / 2}$ are the contours $m_{\chi^{ \pm}}$ $=103.5 \mathrm{GeV}$. The dark shaded region is excluded because a charged particle is lighter than the neutralino, and the darker shaded region is excluded because the LSP is a sneutrino. The light shaded areas are the cosmologically preferred regions with $0.1 \leqslant \Omega{ }_{\chi} h^{2} \leqslant 0.3$ in panels $(\mathrm{a}, \mathrm{b})$ and $0.094 \leqslant \Omega_{\chi} h^{2} \leqslant 0.129$ in panels $(\mathrm{c}, \mathrm{d})$. The dark dot-dashed lines indicate the GUT stability constraint. There are two such lines for each panel and only the areas in between are allowed by this constraint.

cause of the increases in the cross sections with $m_{1 / 2}$, there are in turn upper bounds on the cross sections, that would not be respected if GUT stability were disregarded. Because the $g_{\mu}-2$ constraint would provide even stronger upper bounds on $m_{1 / 2}$, it would also impose stronger upper bounds on the cross sections. In Figs. 1-3, we have left off the $g_{\mu}$ -2 contours to avoid confusion with the cross section con- tours we are highlighting here. For the case with $\mu$ $=400 \mathrm{GeV}$ and $m_{A}=400 \mathrm{GeV}$, the $g_{\mu}-2$ constraint places an upper limit on $m_{1 / 2}$ of about $400 \mathrm{GeV}$ and for the case with $\mu=700 \mathrm{GeV}$ and $m_{A}=700 \mathrm{GeV}$, the limit is $m_{1 / 2}$ $\$ 450 \mathrm{GeV}$.

In panels $(\mathrm{a}, \mathrm{b})$, we display the regions of the planes where $0.1<\Omega_{\chi} h^{2}<0.3$, whereas in panels $(\mathrm{c}, \mathrm{d})$ we restrict to the 

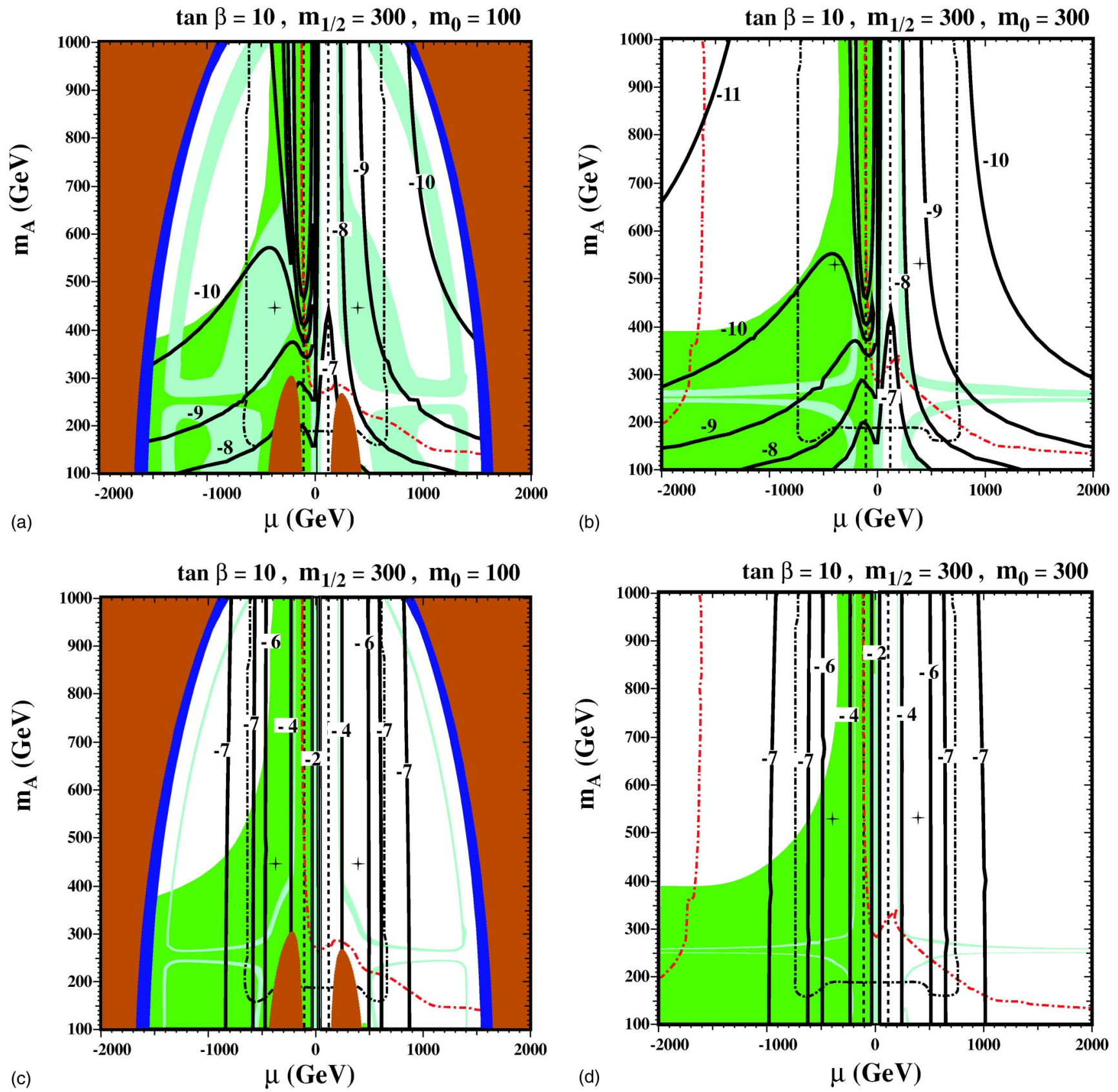

FIG. 2. Contours of $(\mathrm{a}, \mathrm{b})$ the spin-independent and $(\mathrm{c}, \mathrm{d})$ the spin-dependent cross sections (solid black lines) are superimposed on the $\operatorname{NUHM}\left(\mu, m_{A}\right)$ planes for $\tan \beta=10,(\mathrm{a}, \mathrm{c}) m_{0}=100 \mathrm{GeV}$ and $m_{1 / 2}=300 \mathrm{GeV},(\mathrm{b}, \mathrm{d}) m_{0}=300 \mathrm{GeV}$ and $m_{1 / 2}=300 \mathrm{GeV}$. The shadings and line styles are the same as in Fig. 1. In particular, the light shaded areas are the cosmologically preferred regions with $0.1 \leqslant \Omega_{\chi} h^{2} \leqslant 0.3$ in panels (a,b) and $0.094 \leqslant \Omega_{\chi} h^{2} \leqslant 0.129$ in panels (c,d). Here, we see in addition the constraint from $b \rightarrow s \gamma$. The excluded region is medium shaded. The crosses denote the CMSSM points for these choices of $m_{0}$ and $m_{1 / 2}$.

range $0.094<\Omega_{\chi} h^{2}<0.129$ favored when the WMAP data are included [11]. Comparing the cross-section contours and the cosmological ranges in the two sets of panels, we see that the allowed ranges of the cross sections are almost unchanged when the WMAP data are included in this analysis.

As already remarked, the cross sections themselves do not vary greatly with $\tan \beta$, but the interplay of the other constraints is rather $\tan \beta$-dependent. In particular, at large $\tan \beta$ the $g_{\mu}-2$ constraint would not reduce significantly the upper bounds on the cross sections.

\section{B. Examples of $\left(\mu, m_{A}\right)$ planes}

We display in Fig. 2 contours of $(a, b)$ the spinindependent and $(\mathrm{c}, \mathrm{d})$ the spin-dependent elastic scattering cross sections, in the cases $\tan \beta=10$, and $(\mathrm{a}, \mathrm{c}) m_{0}$ $=100 \mathrm{GeV}$ and $m_{1 / 2}=300 \mathrm{GeV}$, (b,d) $m_{0}=300 \mathrm{GeV}$ and $m_{1 / 2}=300 \mathrm{GeV}$. We see that there are large suppressions in the spin-independent cross section for $\mu \sim-100 \mathrm{GeV}$ and $m_{A} \gtrsim 500 \mathrm{GeV}$, reflecting a cancellation in the matrix element. Apart from this, the cross sections generally decrease with increasing $|\mu|$ and (to a lesser extent) $m_{A}$. In this sense, 

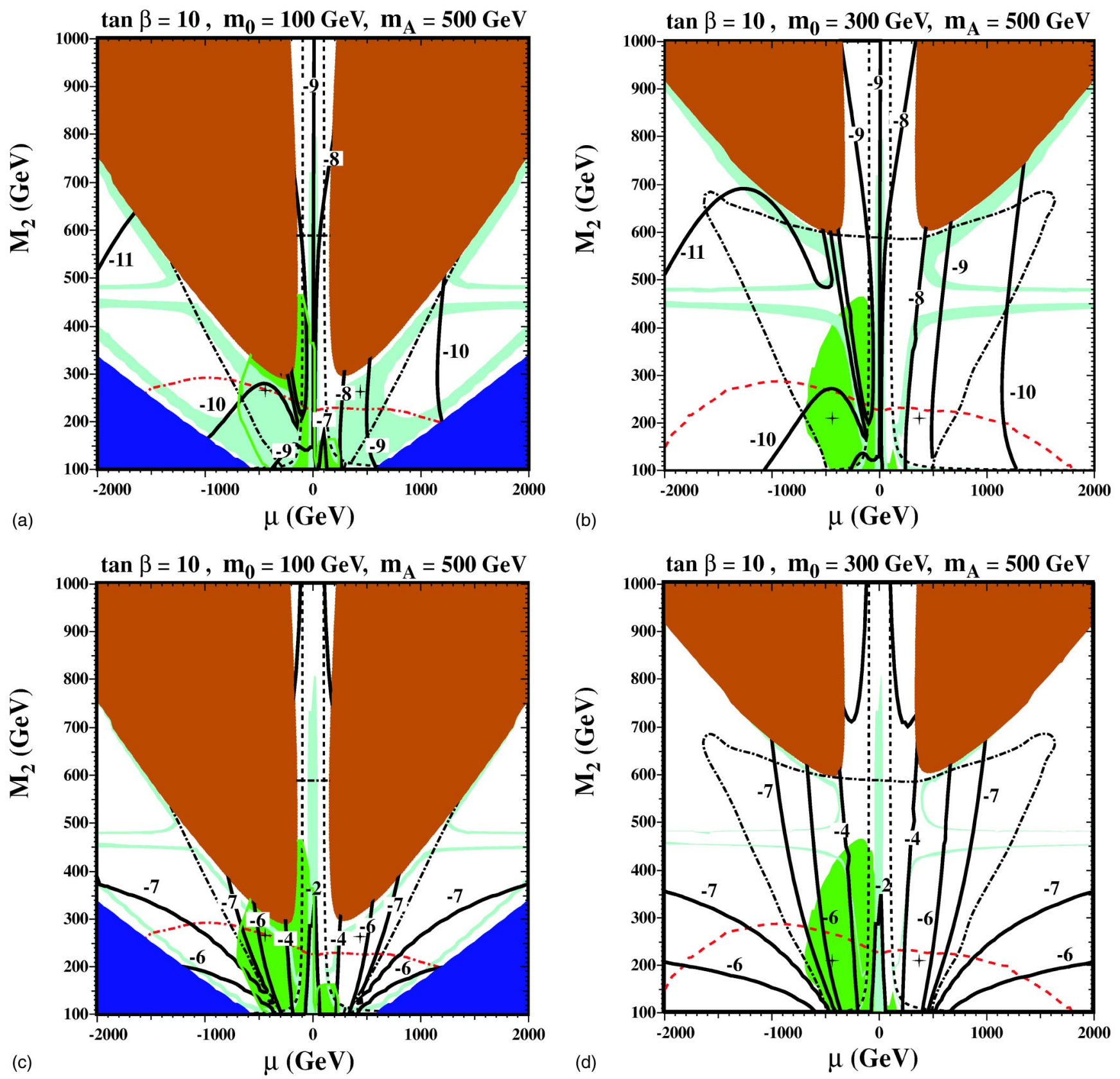

FIG. 3. Contours (a,b) of the spin-independent and (c,d) spin-dependent cross sections (solid black lines) are superimposed on the NUHM $\left(\mu, M_{2}\right)$ planes for $\tan \beta=10,(\mathrm{a}, \mathrm{c}) m_{0}=100 \mathrm{GeV}$ and $m_{A}=500 \mathrm{GeV},(\mathrm{b}, \mathrm{d}) m_{0}=300 \mathrm{GeV}$ and $m_{A}=500 \mathrm{GeV}$. The shadings and line styles are the same as in Fig. 2, the light shaded areas being the cosmologically preferred regions with $0.1 \leqslant \Omega_{\chi} h^{2} \leqslant 0.3$ in panels (a,b) and $0.094 \leqslant \Omega_{\chi} h^{2} \leqslant 0.129$ in panels $(\mathrm{c}, \mathrm{d})$, and the crosses denote the CMSSM points for these choices of $m_{0}$ and $m_{A}$.

the lower bounds on $|\mu|$ and $m_{A}$ set an upper bound on the cross section in the allowed region.

In the CMSSM, cancellations which drive the cross section to extremely small values occur only in the spinindependent case, and only for $\mu<0$. In the NUHM model, however, we find that there is a new source for a cancellation which affects the spin-dependent cross section for either sign of $\mu$. The reason this occurs is as follows. In the CMSSM, the spin-dependent cross section is dominated by the upsquark exchange term and, despite the difference in the relative signs of the up-type and down-type contributions, the total cross section remains reasonably large. On the other hand, in the NUHM case considered here, low-energy sfermion masses are affected by the splitting between the soft supersymmetry-breaking Higgs boson masses at the GUT scale, $S=g_{1}^{2}\left(m_{1}^{2}-m_{2}^{2}\right) / 4$. When $S \neq 0$, the up squarks get somewhat heavier and the down squarks somewhat lighter as $S$ increases (see [17] for details on the effects of $S$ in the renormalization-group equations), opening up the possibility of a cancellation between the contributions. In Fig. 2(c,d), the cancellation occurs between the two $10^{-7} \mathrm{pb}$ contours very close to, but outside the GUT stability curve. The GUT stability requirement, that bounds $|\mu| \lesssim 700 \mathrm{GeV}$ in the figures displayed, therefore provides lower bounds on the spin- 
dependent cross sections. These are somewhat lower than the values found at the CMSSM points indicated by crosses in Fig. 2. ${ }^{5}$ The same cannot be said for the spin-independent cross-sections, because the very small cross sections due to cancellations occur within the GUT stability region, except when one applies the $g_{\mu}-2$ constraint, which excludes the $\mu<0$ region. The interplay of the other constraints is more complicated: as usual, $\mu<0$ is disfavored by the $m_{h}, b$ $\rightarrow s \gamma$ and $g_{\mu}-2$ constraints. The upshot for $\mu>0$ is that the cross sections are bounded above by the $\Omega_{\chi} h^{2}$ constraint, so that they cannot be much more than a factor of $\sim 10$ greater than the CMSSM values.

For the values of parameters chosen in Fig. 2(a,c), we obtain a spin-independent cross-section of $\sigma_{S I}=2.6 \times 10^{-9}$ $\mathrm{pb}$ and a spin-dependent cross-section of $\sigma_{S D}=5.4 \times 10^{-6} \mathrm{pb}$ in the CMSSM for $\mu>0$ (cf. the position of the crosses in the figures). In the NUHM, we find that the range of possible cross sections (for this case) is $3 \times 10^{-10} \mathrm{pb} \lesssim \sigma_{S I} \leqslant 3$ $\times 10^{-8} \mathrm{pb}$ and $3 \times 10^{-8} \mathrm{pb} \lesssim \sigma_{S D} \lesssim 1.6 \times 10^{-4} \mathrm{pb}$ when all constraints other than $g_{\mu}-2$ are included. For the parameters in Fig. 2(b,d), we find the CMSSM spin-independent cross section $\sigma_{S I}=1.9 \times 10^{-9} \mathrm{pb}$, while the spin-dependent cross section is relatively unchanged. Note, however, that this CMSSM point would be excluded due to an excessive value for $\Omega h^{2}(>1)$. In the NUHM, this parameter choice is allowed and gives the range $10^{-9} \mathrm{pb} \lesssim \sigma_{S I} \lesssim 8 \times 10^{-8} \mathrm{pb}$ and $9 \times 10^{-9} \mathrm{pb} \lesssim \sigma_{S D} \lesssim 2 \times 10^{-4} \mathrm{pb}$ for the elastic cross sections. In this case, there is essentially no "bulk" cosmological region, and the spread in $\sigma$ is due to the region where the relic density is due to the heavy Higgs $s$-channel exchange, allowing for a larger range in $\mu$.

As in Fig. 1, the light shaded areas have $0.1 \leqslant \Omega_{\chi} h^{2}$ $\leqslant 0.3$ in panels $(\mathrm{a}, \mathrm{b})$ and the range $0.094 \leqslant \Omega_{\chi} h^{2} \leqslant 0.129$ preferred when WMAP data are included [11] in panels (c,d). Also as in Fig. 1, comparing the cross-section contours and the cosmological regions, we see very little change in the allowed ranges of the cross sections when WMAP is included.

\section{Examples of $\left(\boldsymbol{\mu}, M_{2}\right)$ planes}

We display in Fig. 3 contours of $(a, b)$ the spinindependent and $(\mathrm{c}, \mathrm{d})$ the spin-dependent elastic scattering cross sections, in the cases $\tan \beta=10$, and $(\mathrm{a}, \mathrm{c}) m_{0}$ $=100 \mathrm{GeV}$ and $m_{A}=500 \mathrm{GeV}$, (b,d) $m_{0}=300 \mathrm{GeV}$ and $m_{A}=500 \mathrm{GeV}$. Because the cross sections vary relatively rapidly, we have not included all the decade cross-section contours in panels $(\mathrm{c}, \mathrm{d})$.

We see again in this case the suppression in the spinindependent cross section for $\mu \sim-100 \mathrm{GeV}$ and $M_{2}$ $\geq 200 \mathrm{GeV}$, apart from which the cross sections decrease with increasing $|\mu|$, at least within the GUT stability region. The spin-dependent cross section, on the other hand, starts rising again at large $|\mu|$, reflecting the fact that one has tra-

\footnotetext{
${ }^{5}$ Analogous crosses did not appear in Fig. 1, just because their positions would lie outside the ranges of parameters plotted in the figures.
}

versed a cancellation in the scattering matrix element of the type described in the previous subsection. Once again, the cancellation is found between the $10^{-7} \mathrm{pb}$ contours just outside the GUT stability curve.

For the values of parameters chosen in Fig. 3(a,c), we obtain a spin-independent cross section $\sigma_{S I}=2 \times 10^{-9} \mathrm{pb}$ and a spin-dependent cross section $\sigma_{S D}=3 \times 10^{-6} \mathrm{pb}$ in the CMSSM (cf. the position of the crosses in the figures). In the NUHM, we find that the range of possible cross sections for this case is $2.5 \times 10^{-11} \mathrm{pb} \lesssim \sigma_{S I} \leqslant 9 \times 10^{-8} \mathrm{pb}$ and $1.5 \times 10^{-9} \mathrm{pb} \lesssim \sigma_{S D} \leqslant 2 \times 10^{-3} \mathrm{pb}$. For the parameters in Fig. 2(b,d), the CMSSM spin-independent cross section is $\sigma_{S I}=3 \times 10^{-9} \mathrm{pb}$, while the spin-dependent cross section is $\sigma_{S D}=10^{-5} \mathrm{pb}$. Note, however, that this CMSSM point would be excluded due to a excessive value for $\Omega h^{2}(\gtrsim 1)$. In the NUHM, this parameter choice is allowed and gives the range $10^{-13} \mathrm{pb} \lesssim \sigma_{S I} \leqslant 2 \times 10^{-8} \mathrm{pb}$ and $9 \times 10^{-9} \mathrm{pb} \lesssim \sigma_{S D}$ $\leqslant 2 \times 10^{-4} \mathrm{pb}$ for the elastic cross sections.

As in Figs. 1 and 2, the light shaded areas in panels $(a, b)$ have $0.1 \leqslant \Omega_{\chi} h^{2} \leqslant 0.3$ in panels $(\mathrm{a}, \mathrm{b})$ and the range 0.094 $\leqslant \Omega_{\chi} h^{2} \leqslant 0.129$ preferred when WMAP data are included [11] in panels (c,d). Also as in the previous figures, comparing the cross-section contours and the cosmological regions, we see very little change in the allowed ranges of the cross sections when WMAP is included. For this reason, the plots in the following sections allow for the full range 0.1 $<\Omega_{\chi} h^{2}<0.3$.

\section{ALLOWED RANGES OF ELASTIC CROSS SECTIONS}

Following our discussion in the previous section of some important features in a few examples of parameter planes in the NUHM, we now display the ranges of elastic scattering cross sections permitted by various theoretical and experimental constraints. We start with the specific NUHM parameter planes discussed above, and then go on to generalize the discussion. In each of the specific planes, we show the effect on the allowed cross section when the phenomenological and cosmological constraints are applied successively. We start with the very simple requirement that the LSP be a neutralino with $m_{\chi^{ \pm}} \gtrsim 103.5 \mathrm{GeV}$ and $m_{e} \gtrsim 99 \mathrm{GeV}$. We then apply either the Higgs cut or the $b \rightarrow s \gamma$ cut. Our standard cut is defined to include these two in addition to the appropriate value for $\Omega h^{2}$. Following the standard cut we apply sequentially the GUT stability constraint and the constraint due to $g_{\mu}-2$.

\section{A. Specific planes}

We consider first the $\left(m_{1 / 2}, m_{0}\right)$ plane for $\tan \beta=10$ and $\mu=m_{A}=400 \mathrm{GeV}$ that was displayed earlier in Fig. 1(a,c). The horizontal axes in the various panels of Fig. 4 correspond to the LSP mass $m_{\chi}$, and the vertical axes show the ranges of $(\mathrm{a}, \mathrm{c}, \mathrm{e})$ the spin-independent and $(\mathrm{b}, \mathrm{d}, \mathrm{f})$ the spindependent elastic cross sections. The first row of panels $(\mathrm{a}, \mathrm{b})$ shows the ranges allowed by our cuts on the LSP (dark lines), $m_{h}$ (lighter lines) and standard cut (shaded), the second row (c,d) displays the further impact of the GUT stability constraint, and the third row $(\mathrm{e}, \mathrm{f})$ implements all the cuts, including that on $g_{\mu}-2$. 

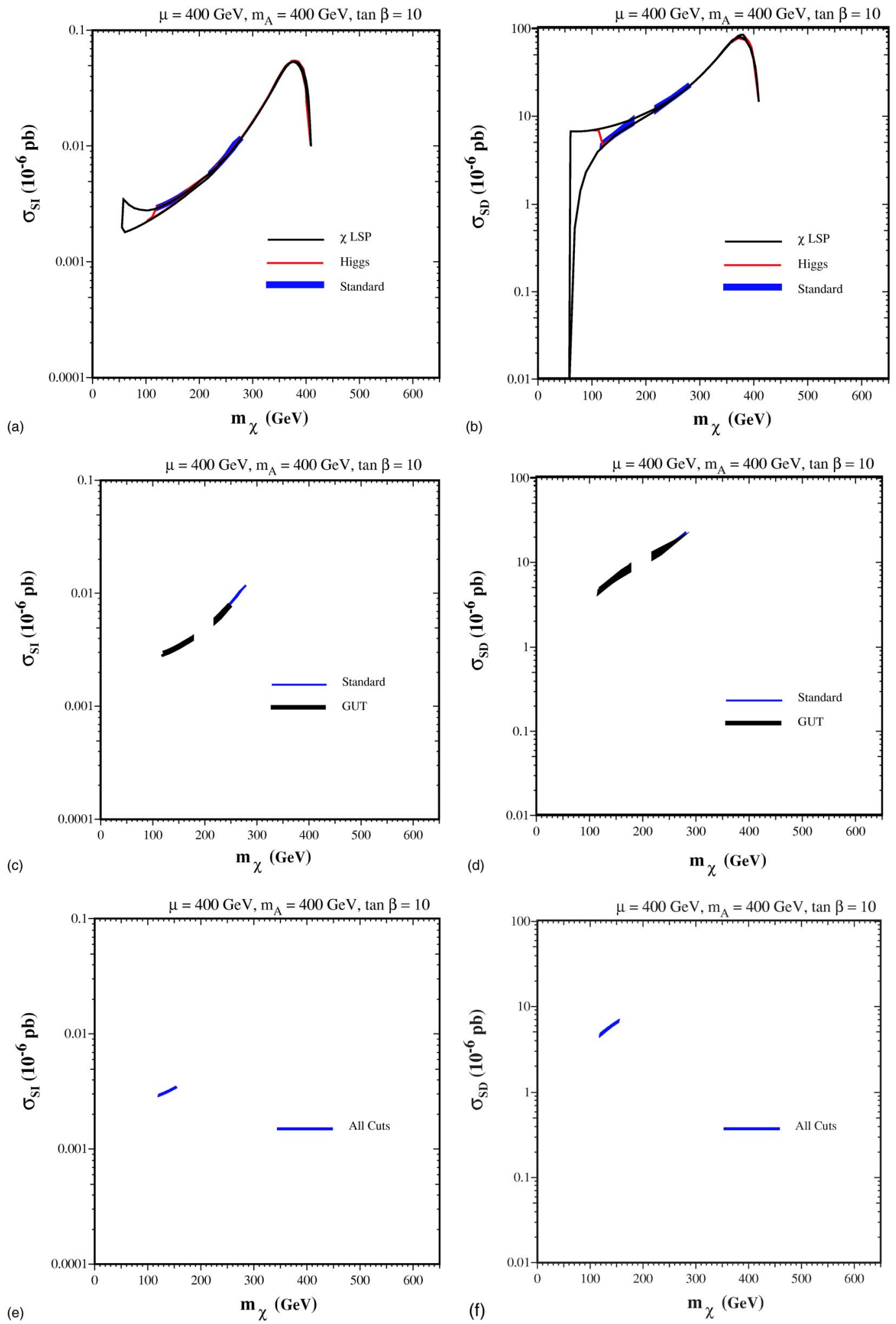

FIG. 4. Cross sections allowed in a slice through the NUHM parameter space provided by the $\left(m_{1 / 2}, m_{0}\right)$ plane for tan $\beta=10$ and $\mu$ $=m_{A}=400 \mathrm{GeV}$. Panels (a,b) are with our cuts on the LSP (dark lines), $m_{h}$ (lighter lines) and standard cut (shaded), panels (c,d) also have the GUT stability constraint imposed, and panels (e,f) apply all cuts, including the possible $g_{\mu}-2$ constraint. The right (left) panels show the spin-(in)dependent cross section, plotted against $m_{\chi}$. 
We see in Fig. 4(a,b) that the scattering cross sections rise monotonically with $m_{\chi}$, except close to the upper limit on $m_{\chi}$, reflecting the increase with $m_{1 / 2}$ that was already commented in connection with Fig. 1. We also note that the ranges of cross sections allowed for any fixed value of $m_{\chi}$ are very restricted, reflecting the fact the the contours of equal cross section in Fig. 1(a,c) are almost vertical in the parameter range of interest. The break that appears in the middle of Fig. 4(a,b), and is seen more clearly in $(\mathrm{c}, \mathrm{d})$, reflects the range of $m_{1 / 2} \sim 500 \mathrm{GeV}$ where $\Omega_{\chi} h^{2}$ is suppressed below the preferred cosmological range by rapid directchannel $\chi \chi$ annihilation via the $H, A$ poles.

The most relevant effect of the extra GUT and $g_{\mu}-2$ constraints is to reduce the range of $m_{1 / 2}$ and hence $m_{\chi}$. As we see in Fig. 4(c,d), the GUT stability constraint removes the points in this NUHM parameter plane that have the largest elastic scattering cross sections. Finally, as we see in Fig. 4(e,f), the $g_{\mu}-2$ constraint confines our attention to points in the NUHM parameter below the rapid-annihilation channel. In this particular case, there is a narrow preferred range of the spin-independent cross section around $3 \times 10^{-9} \mathrm{pb}$, and the preferred range of the spin-dependent cross section is around $6 \times 10^{-6} \mathrm{pb}$.

Our second example is the $\left(\mu, m_{A}\right)$ plane for $\tan \beta$ $=10, m_{1 / 2}=300 \mathrm{GeV}$ and $m_{0}=100 \mathrm{GeV}$, displayed previously in panels (a,c) of Fig. 2. As seen in Fig. 5(a,b), when one imposes the standard $\Omega_{\chi} h^{2}, m_{h}$ and $b \rightarrow s \gamma$ constraints, the cross sections generally decrease with $m_{\chi}$. However, in the spin-dependent case the cross section reaches a locus of near zeros, after which it rises again. These cancellations are avoided in this case when the Higgs and $b \rightarrow s \gamma$ constraints are applied. As then seen in Fig. 5(c,d), the GUT stability constraint, which removes portions of this NUHM parameter plane at large $|\mu|$ and small $m_{A}$, strengthens in this case the lower bounds on the cross sections. The effect of the $g_{\mu}$ -2 constraint is less marked in this case, as seen in Fig. $5(\mathrm{e}, \mathrm{f})$. The final allowed ranges of the cross section are considerably wider than in the previous example: $\sim 10^{-9}$ to $\sim 10^{-8} \mathrm{pb}$ in the spin-independent case and $\sim 10^{-7}$ to $\sim 10^{-4} \mathrm{pb}$ in the spin-dependent case.

Our final example is the $\left(\mu, M_{2}\right)$ plane for $\tan \beta$ $=10, m_{0}=100 \mathrm{GeV}$ and $m_{A}=500 \mathrm{GeV}$, shown in Fig. 6. In this case, the standard cuts allow a particularly wide range of cross sections, varying by infinite (over 3 ) orders of magnitude for the spin-(in)dependent case. The different regions allowed by the standard cuts reflect the different branches of parameter space in Fig. 3. We note that some of these branches are due to the differences between positive and negative $\mu$. We also note that some of the boundaries are due to our imposed cutoff of $|\mu| \leqslant 2 \mathrm{TeV}$. In particular, had we allowed for larger values of $|\mu|$, we would have found larger neutralino masses, and the lower bounds for both the Higgs and $b \rightarrow s \gamma$ cuts (which differ for positive and negative $\mu$ ) would also have been lowered. The general tendency of the standard cuts is to decrease the cross sections with increasing $m_{\chi}$, though with considerable variation. The GUT stability constraint in this case removes a region at large $M_{2}$ and hence $m_{\chi}$, that removes the points with the lowest cross sections. This effect is particularly marked for the spindependent case, where the range is now reduced to (a mere) 5 orders of magnitude. The $g_{\mu}-2$ constraint further raises the lower bounds on the cross sections, so that they vary through just over 3 (under 2) orders of magnitude in the spin-(in)dependent case.

Notice that there is an isolated point at $m_{\chi} \sim 80 \mathrm{GeV}$. This corresponds to a very narrow region just to the right of the chargino mass-bound line in Fig. 3(a,c) at $\mu$ $\sim 120 \mathrm{GeV}, M_{2} \sim 240 \mathrm{GeV}$, which is not visible because of the plotting resolution. What happens is that as $\mu$ decreases, $m_{\chi}$ falls below the $m_{h}$ threshold, and hence the annihilation cross section decreases leading to an acceptable value of $\Omega_{\chi} h^{2}$. However, as $\mu$ decreases further, neutralino-chargino coannihilation becomes stronger, suppressing $\Omega_{\chi} h^{2}$ again to be less than 0.1 .

This brief survey shows the importance of implementing correctly the GUT stability constraint, which may (in different cases) bound the cross sections either above or below. As many authors have previously pointed out in the CMSSM case, the $g_{\mu}-2$ constraint is also potentially important. In certain cases, it can also strengthen significantly the lower limits on the NUHM cross sections.

\section{B. General analysis}

Equipped with the above information about some specific examples, we now make a general analysis of the possible values of the elastic scattering cross sections. In the first place, we concentrate on the case $\tan \beta=10$, but relaxing the previous restricted choices of other parameters that we took as examples. To produce the plots, we generate random points (about 30000 points for each plot) within the following ranges:

$$
\begin{array}{r}
100 \mathrm{GeV} \leqslant m_{1 / 2} \leqslant 1500 \mathrm{GeV}, \\
0 \leqslant m_{0} \leqslant 1000 \mathrm{GeV}, \\
-2000 \mathrm{GeV} \leqslant \mu \leqslant 2000 \mathrm{GeV}, \\
90 \mathrm{GeV} \leqslant m_{A} \leqslant 1500 \mathrm{GeV} .
\end{array}
$$

We first impose the same standard experimental and phenomenological constraints discussed earlier, namely: a consistent electroweak vacuum, $0.1<\Omega_{\chi} h^{2}<0.3, m_{h}$ $>114 \mathrm{GeV}$ and the $b \rightarrow s \gamma$ constraint. In a previous paper on the CMSSM [4], we rescaled the elastic scattering cross sections for models that predicted $\Omega_{\chi} h^{2}<0.1$ by the factor $\Omega_{\chi} h^{2} / 0.1$, so as to account for the fact that the neutralino could constitute at most this fraction of the galactic halo. In the results that follow, we show how this rescaling affects the upper limits on the cross sections. Next we apply the GUT stability cut, and finally we show the implications of imposing the cut on $g_{\mu}-2$. The resulting ranges of the elastic scattering cross sections for $\tan \beta=10$ are displayed in Fig. 7.

We note that the spin-independent cross section shown in Fig. 7(a) may be as large as a few $\times 10^{-8} \mathrm{pb}$, decreasing only slightly as $m_{\chi}$ increases, while values lower than $10^{-13}$ 

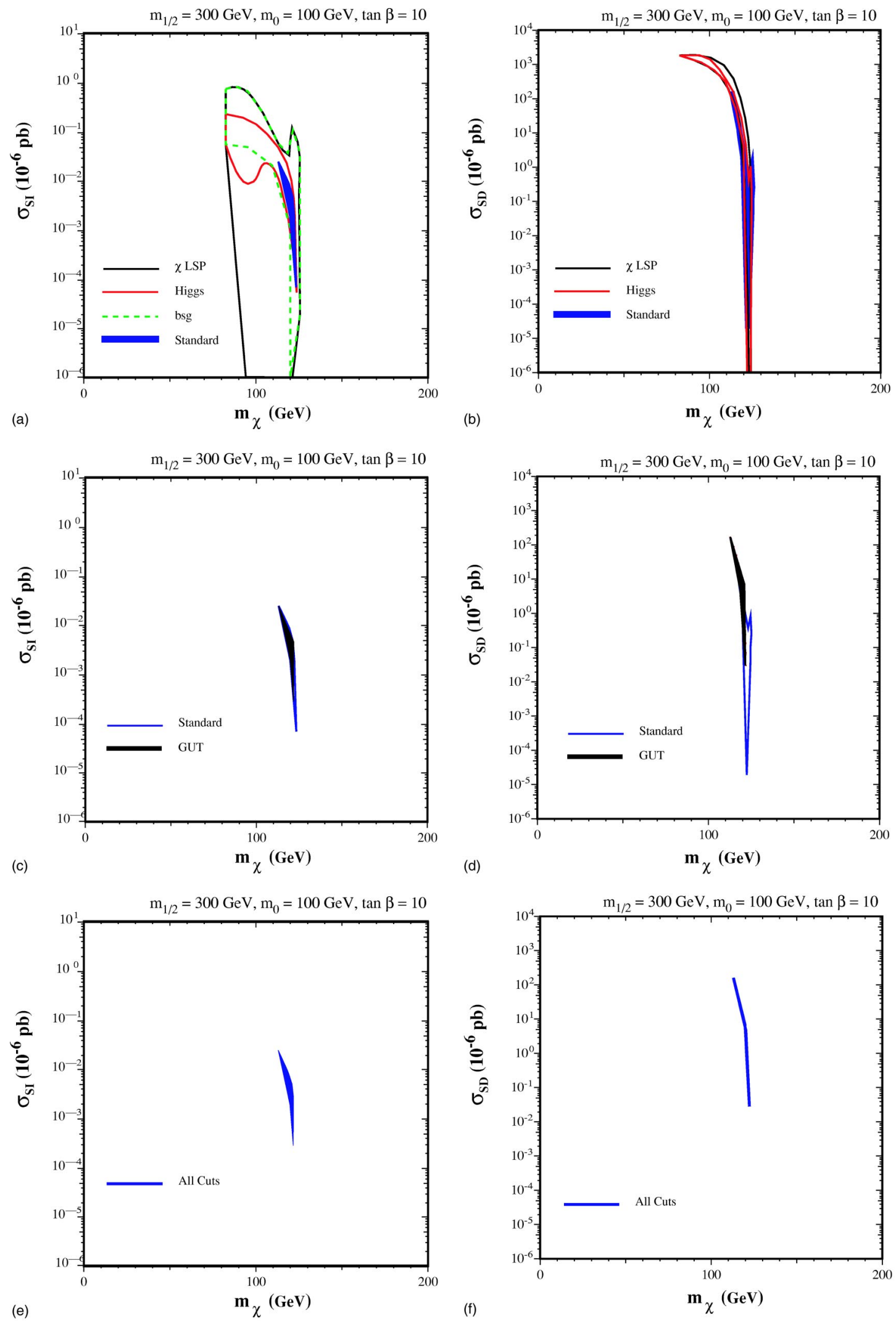

FIG. 5. Cross sections allowed in a slice through the NUHM parameter space provided by the $\left(\mu, m_{A}\right)$ plane for tan $\beta=10$ and $m_{1 / 2}$ $=300 \mathrm{GeV}, m_{0}=100 \mathrm{GeV}$. The selections of points in the different panels are the same as in Fig. 4, as are their indications. In addition, panel (a) also shows the $b \rightarrow s \gamma$ cut (dashed lines). 

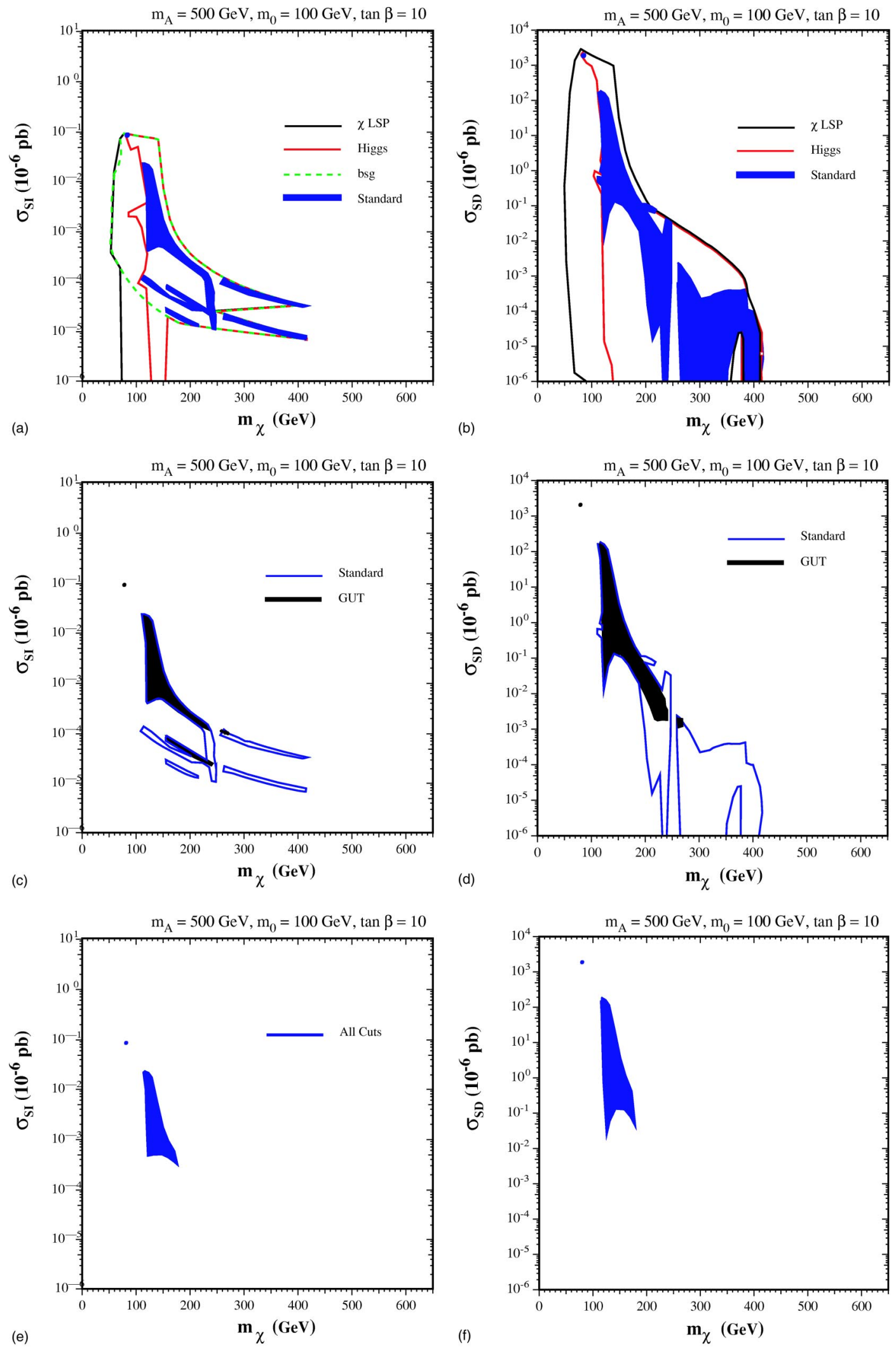

FIG. 6. Cross sections allowed in a slice through the NUHM parameter space provided by the $\left(\mu, M_{2}\right)$ plane for tan $\beta=10$ and $m_{0}$ $=100 \mathrm{GeV}, m_{A}=500 \mathrm{GeV}$. The selections of points in the different panels are the same as in Fig. 5, as are their indications. 

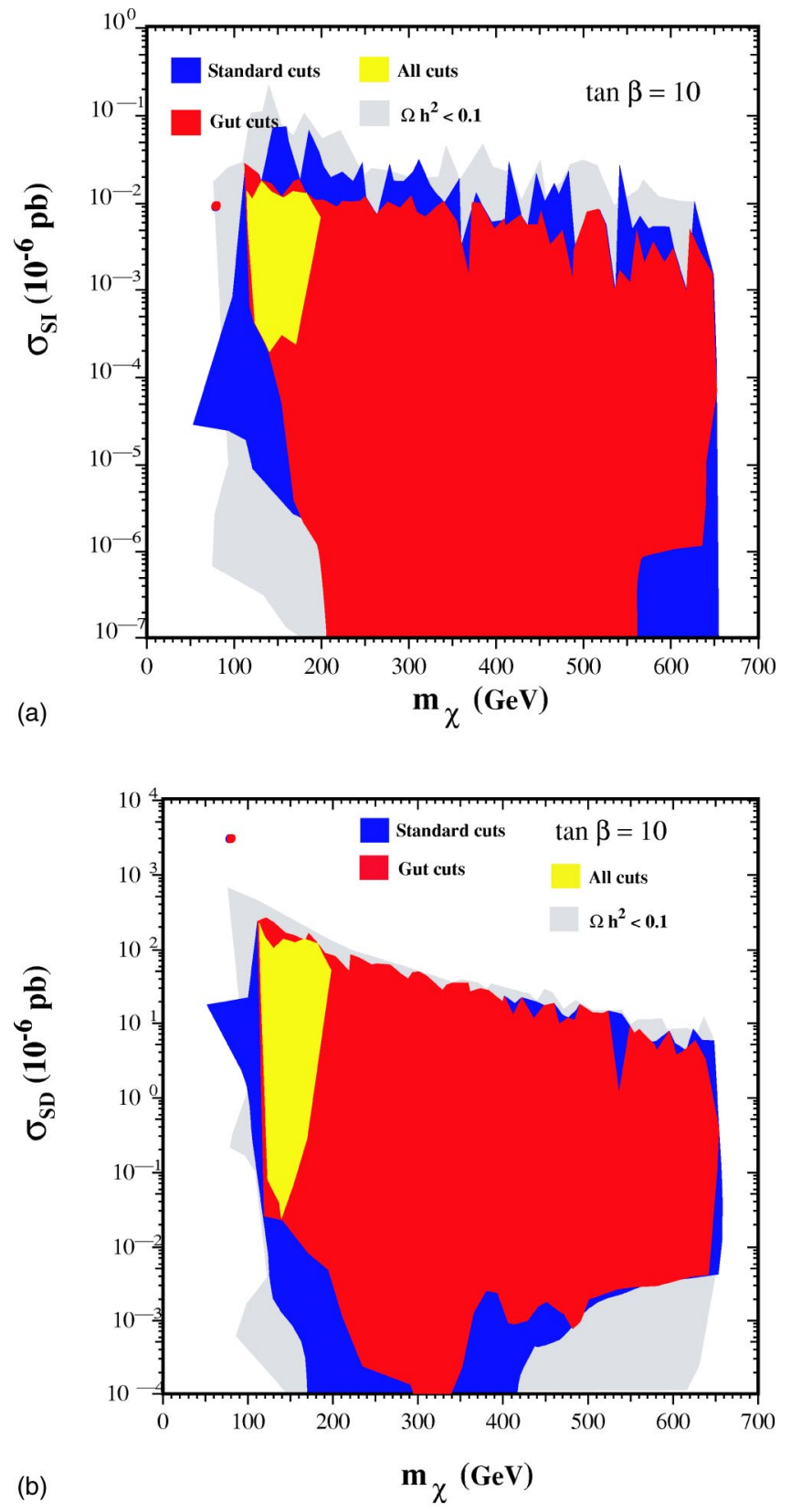

FIG. 7. Ranges of (a) the spin-independent and (b) the spindependent cross sections for $\tan \beta=10$. The ranges allowed by the standard cuts on $\Omega_{\chi} h^{2}, m_{h}$ and $b \rightarrow s \gamma$ have dark shading, those still allowed by the GUT stability cut have medium shading, and those still allowed after applying all the cuts including $g_{\mu}-2$ have light shading. The pale shaded region corresponds to the extra area of points with low relic densities, whose cross sections have been rescaled appropriately.

$\mathrm{pb}$ cannot be excluded. The raggedness of the upper limit on the cross section reflects the fact that our sampling produced very few points with such large cross sections: values in between the crags cannot be excluded, but must be very rare. Between the crags there are valleys, below which the density of points is significantly larger. The lowest values of the cross section occur for $\mu<0$, where cancellations are possible in the spin-independent scattering matrix element, as discussed in a previous paper [3]. The GUT stability constraints exclude some low cross-section values at both small and large $m_{\chi}$, but do not provide an overall lower bound. It does however, lower the upper bound by as much as a factor of about 5. Negative $\mu$, and hence very low cross-section values, would be excluded by the putative $g_{\mu}-2$ constraint, as shown in Fig. 7(a). For $\mu>0$, we find (not shown) spinindependent cross sections only above $10^{-11} \mathrm{pb}$.

Figure 7(a) also displays the region (pale shaded) which survives all standard cuts, except that $\Omega h^{2}<0.1$. For points in this region, we have rescaled the cross section by a factor of $\Omega h^{2} / 0.1$, to allow for the fact that the LSP could not in this case make up all the cold dark matter in the Universe, and hence a fortiori in the galactic halo. As one can see, many of the spaces between the crags are now filled in with such points, but very few give significantly larger cross sections.

In the case of the spin-dependent cross section shown in Fig. 7(b), the upper limit is better defined, and decreases monotonically from $\sim 3 \times 10^{-3} \mathrm{pb}$ for $m_{\chi} \sim 80 \mathrm{GeV}$ to $\sim 5$ $\times 10^{-6} \mathrm{pb}$ for $m_{\chi} \sim 650 \mathrm{GeV}$. Cross sections lower than $\sim 10^{-10} \mathrm{pb}$ are possible for either sign of $\mu$, even after imposing the GUT stability cuts. In this case, the points with rescaled cross sections enhance the cross section by a factor of about 3 at low neutralino masses.

The isolated point in both panels at $m_{\chi} \sim 80 \mathrm{GeV}$ now corresponds to a narrow region around $\mu \sim-110 \mathrm{GeV}$ and large $m_{A}>1000 \mathrm{GeV}$, which is between the $m_{h}=114 \mathrm{GeV}$ and $m_{\chi^{ \pm}}=103.5 \mathrm{GeV}$ lines. Its existence is very sensitive to the implementation of the Higgs boson mass bound.

We note that the ranges allowed by $g_{\mu}-2$ are relatively restricted. For a start, we find that $110 \mathrm{GeV} \lesssim m_{\chi}$ $\$ 200 \mathrm{GeV}$. Moreover, even within this range, very low cross-section values are excluded. Overall, we find ranges between $\sim 2 \times 10^{-8} \mathrm{pb}$ and $\sim 2 \times 10^{-10} \mathrm{pb}$ for the spinindependent cross section, and between $\sim 2 \times 10^{-4} \mathrm{pb}$ and $\sim 2 \times 10^{-8} \mathrm{pb}$ for the spin-independent cross section.

Ranges for the spin-independent and -dependent cross sections for $\tan \beta=20$ are shown in Fig. 8. Looking first at the spin-independent cross section in panel (a), we see that our standard cuts on $m_{h}, \Omega_{\chi} h^{2}$ and $b \rightarrow s \gamma$ would allow somewhat larger values than for $\tan \beta=10$. This difference is less marked when the GUT stability cut is also applied, except for some exceptional parameter choices at small $m_{\chi}$. The jaggedness of the peaks is more pronounced at this value of $\tan \beta$. Once again, we emphasize that while we do not expect the area between the peaks to be empty, the density of points there is extremely low. When one keeps the low-relicdensity points (rescaled appropriately), we see that indeed the crags are filled in to some extent. For $\tan \beta=20$ these points do not enhance the cross section significantly. The values of $m_{\chi}$ allowed by $g_{\mu}-2$ are larger for $\tan \beta=20$ than for $\tan \beta=10$, and lower cross sections are also attainable. Overall, the spin-independent cross section may vary between $\sim 3 \times 10^{-7} \mathrm{pb}$ and $10^{-10} \mathrm{pb}$ when $\tan \beta=20$.

In the case of the spin-dependent cross section shown in Fig. 8(b), the upper and lower bounds for $\tan \beta=20$ are very similar to those for $\tan \beta=10$ if only the standard and GUT 

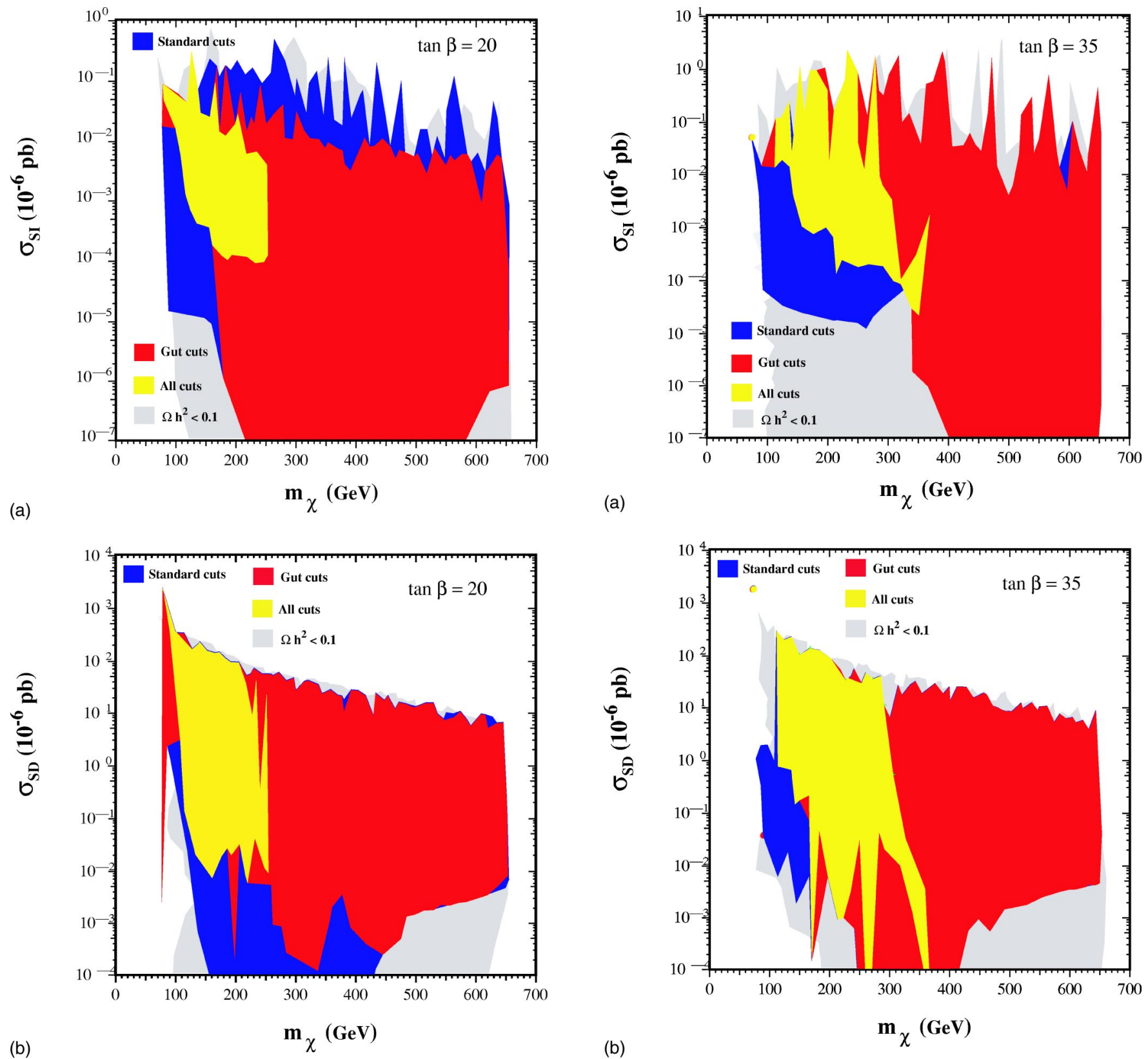

FIG. 8. Ranges of (a) the spin-independent and (b) the spindependent cross sections for $\tan \beta=20$. The shadings are the same as in Fig. 7.

cuts are applied. However, slightly lower values of the cross section are allowed when the $g_{\mu}-2$ constraint is applied, without a strong dependence on the value of $m_{\chi}$ (which may be somewhat larger than in the case of $\tan \beta=10$ ). For $\tan \beta=20$, the spin-dependent cross section ranges between $\sim 2 \times 10^{-3} \mathrm{pb}$ and $\sim 10^{-8} \mathrm{pb}$.

Continuing now to $\tan \beta=35$, as shown in Fig. 9, we see again that the spin-independent cross section may be somewhat larger still than for $\tan \beta=20$, though the same is not true for the spin-dependent cross sections. Once again, the $g_{\mu}-2$ constraint allows larger values of $m_{\chi}$ as $\tan \beta$ is increased, opening up the possibility of a smaller cross section, particularly in the spin-dependent case where a cancellation

FIG. 9. Ranges of (a) the spin-independent and (b) the spindependent cross sections for $\tan \beta=35$. The shadings are the same as in Fig. 7.

may occur, potentially suppressing the cross section by a couple of orders of magnitude. Both these tendencies are accentuated in the case $\tan \beta=50$, as shown in Fig. 10. For both $\tan \beta=35,50$, the spin-independent cross section may, in some exceptional cases, rise above $10^{-6} \mathrm{pb}$, even after implementing all the cuts. It may also drop as low as $10^{-10}$ $\mathrm{pb}$. The maximal spin-dependent cross section is above $10^{-4}$ $\mathrm{pb}$ in the $\tan \beta=35$ case, and somewhat below $10^{-4} \mathrm{pb}$ in the $\tan \beta=50$ case. In both cases, it may also drop as low as $10^{-10} \mathrm{pb}$.

Finally, in Fig. 11, we display the allowed ranges of (a) the spin-independent and (b) the spin-dependent cross sections when we sample randomly $\tan \beta$ as well as the other 

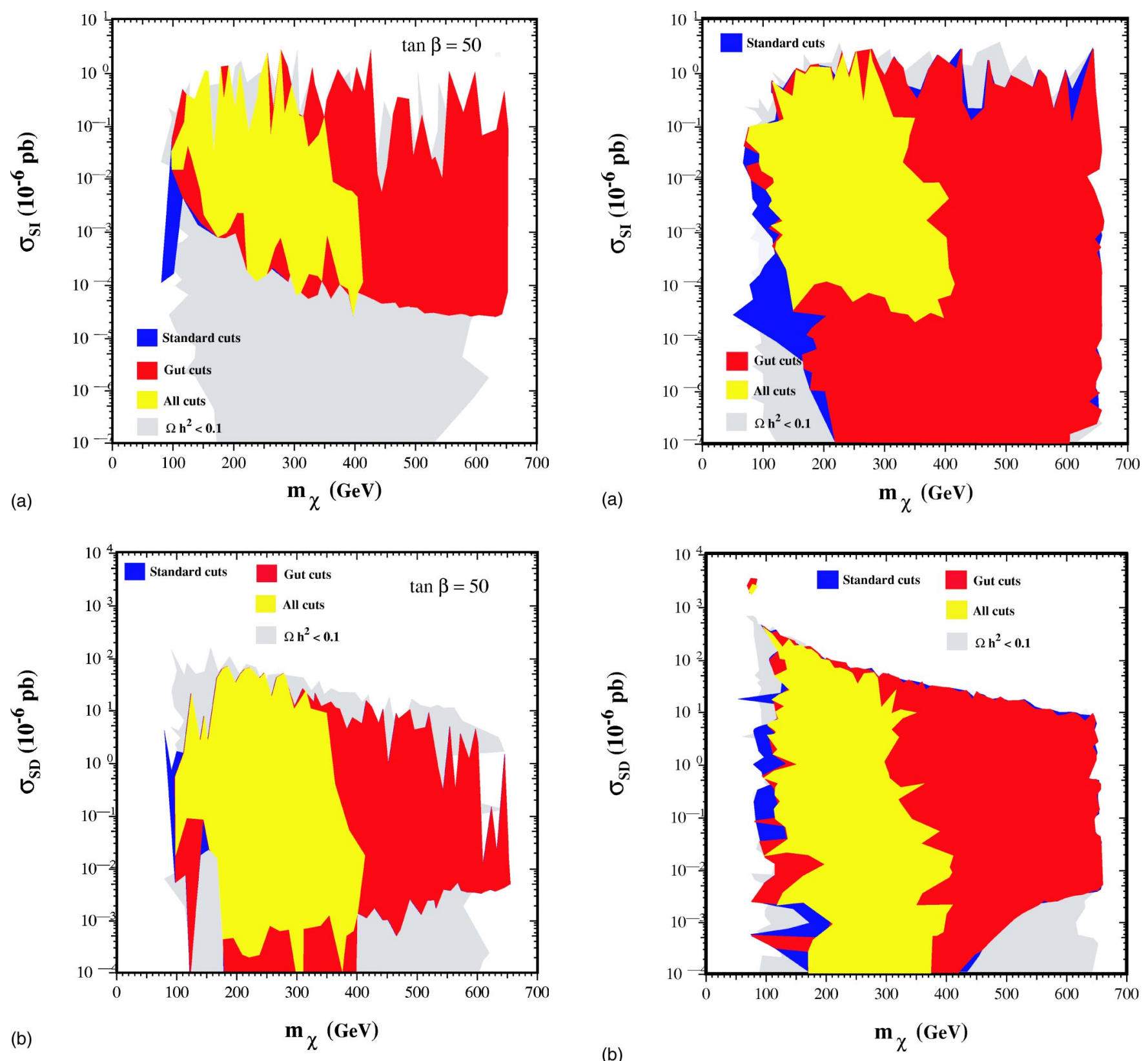

FIG. 10. Ranges of (a) the spin-independent and (b) the spindependent cross sections for $\tan \beta=50$. The shadings are the same as in Fig. 7.

NUHM parameters. We see no big surprises compared with the previous plots for individual values of $\tan \beta$, but observe that the boundaries of the shaded regions are very ragged, reflecting the finite sample size. After incorporating all the cuts, including that motivated by $g_{\mu}-2$, we find that the spin-independent cross section has the range $10^{-6} \mathrm{pb} \gtrsim \sigma_{S I}$ $\gtrsim 10^{-10} \mathrm{pb}$, and the spin-dependent cross section has the range $10^{-4} \mathrm{pb} \gtrsim \sigma_{S D} \gtrsim 10^{-10} \mathrm{pb}$, with somewhat larger (smaller) values being possible in exceptional cases. If the $g_{\mu}-2$ cut is removed, the upper limits on the cross sections are unchanged, but much lower values become possible: $\sigma_{S I} \ll 10^{-13} \mathrm{pb}$ and $\sigma_{S D} \ll 10^{-10} \mathrm{pb}$.

FIG. 11. Ranges of (a) the spin-independent and (b) the spindependent cross sections, sampling randomly all allowed values of $\tan \beta$. The shadings are the same as in Fig. 7.

\section{CONCLUSIONS}

We have discussed in this paper the possible ranges of spin-(in)dependent elastic cross sections in the NUHM, in which the input soft supersymmetry-breaking masses of the Higgs doublets are allowed to differ from those of the squarks and sleptons-which are still assumed to be universal. Figure 11 summarizes the results, including the flexibility of varying $\tan \beta$ as well as the input scalar and fermion masses $m_{0,1 / 2}$, the Higgs mixing parameter $\mu$ and the pseudoscalar Higgs boson mass $m_{A}$. In this paper, we have not varied the trilinear soft supersymmetry-breaking parameter $A$, whose effective low-energy value has in any case a limited range when renormalized from the GUT scale down- 
wards, so that it does not have a large effect on the cross sections we study.

We have stressed in this paper the importance of incorporating consistently all the available phenomenological constraints from laboratory experiments and cosmology. We have also stressed the importance of taking into account the running of the NUHM parameters over the full range of scales between the GUT scale and the electroweak scale. As we have discussed explicitly, the requirement that the effective scalar potential be stable at the GUT scale restricts the allowed variations in the non-universalities of the soft supersymmetry-breaking Higgs boson masses.

The effects of our phenomenological cuts and this GUT stability requirement can be seen in Fig. 11, and also in the previous Figs. 7, 8, 9 and 10 for $\tan \beta=10,20,35$ and 50, respectively. Some examples of our analysis for specific slices through the NUHM parameter space can be seen in earlier figures.

In general, we find that cross sections may differ by a few orders of magnitude from those found in the CMSSM, in which the soft supersymmetry-breaking Higgs boson masses are assumed to be universal with the slepton and squark masses at the GUT scale. However, the spin-independent cross section normally lies well below the present experimental sensitivity. Only in a few exceptional cases do we find a cross section as large as the present experimental sensitivity $\sigma_{S I} \sim 10^{-6} \mathrm{pb}$, and a sensitivity $\sigma_{S I} \sim 10^{-10} \mathrm{pb}$ would be required to cover most of the preferred domain of NUHM parameter space. Even this sensitivity would be insufficient if one disregards the indication from $g_{\mu}-2$, which is the only constraint that motivates a useful upper bound on the sparticle mass scale, and hence a useful lower bound on $\sigma_{S I}$. In the case of the spin-dependent cross section, values of $\sigma_{S D}$ as low as $\sim 10^{-10} \mathrm{pb}$ cannot be excluded even if one takes seriously the $g_{\mu}-2$ constraint.

The next logical step in the exploration of the MSSM, relaxing further the assumption of full scalar-mass universality as in the CMSSM, is to allow the soft supersymmetrybreaking slepton and squark masses to differ at the GUT scale. The allowed ranges of the effective low-energy slepton and squark masses, after renormalization, will be restricted by analogues of the GUT stability constraints we have applied in this paper. In particular, we note that general choices of the effective low-energy slepton and squark masses may lead (in particular) to tachyonic squarks below the GUT scale, when renormalized to higher scales. We will explore in future publications the effects on the parameter space and the ranges of cross sections of applying consistently the GUT stability constraints to the general nonuniversal MSSM.

\section{ACKNOWLEDGMENTS}

The work of K.A.O. and Y.S. was supported in part by DOE grant DE-FG02-94ER-40823.
[1] K. Griest, Phys. Rev. D 38, 2357 (1988); J. Ellis and R. Flores, Nucl. Phys. B307, 883 (1988); R. Barbieri, M. Frigeni, and G. Giudice, ibid. B313, 725 (1989); R. Flores, K. A. Olive, and M. Srednicki, Phys. Lett. B 237, 72 (1990); J. Ellis and R. Flores, ibid. 263, 259 (1991); ibid. 300, 175 (1993); M. Drees and M. M. Nojiri, Phys. Rev. D 48, 3483 (1993); V. Bednyakov, H. V. Klapdor-Kleingrothaus, and S. Kovalenko, ibid. 50, 7128 (1994); R. Arnowitt and P. Nath, ibid. 54, 2374 (1996); L. Bergstrom and P. Gondolo, Astropart. Phys. 5, 263 (1996); H. Baer and M. Brhlik, Phys. Rev. D 57, 567 (1998); A. Corsetti and P. Nath, ibid. 64, 125010 (2001).

[2] E. Accomando, R. Arnowitt, B. Dutta, and Y. Santoso, Nucl. Phys. B585, 124 (2000).

[3] J. Ellis, A. Ferstl, and K. A. Olive, Phys. Lett. B 481, 304 (2000).

[4] J. Ellis, A. Ferstl, and K. A. Olive, Phys. Rev. D 63, 065016 (2001).

[5] J. R. Ellis, J. L. Feng, A. Ferstl, K. T. Matchev, and K. A. Olive, Eur. Phys. J. C 24, 311 (2002).

[6] J. R. Ellis, A. Ferstl, and K. A. Olive, Phys. Lett. B 532, 318 (2002).

[7] For other scattering calculations, see, for example, J. L. Feng, K. T. Matchev, and F. Wilczek, Phys. Lett. B 482, 388 (2000); M. Drees, Y. G. Kim, T. Kobayashi, and M. M. Nojiri, Phys. Rev. D 63, 115009 (2001); Y. G. Kim and M. M. Nojiri, Prog. Theor. Phys. 106, 561 (2001); A. B. Lahanas, D. V. Nanopoulos, and V. C. Spanos, Mod. Phys. Lett. A 16, 1229 (2001); A. B. Lahanas, D. V. Nanopoulos, and V. C. Spanos, Phys. Lett. B
518, 94 (2001); E. A. Baltz and P. Gondolo, Phys. Rev. Lett. 86, 5004 (2001); Y. G. Kim, T. Nihei, L. Roszkowski, and R. Ruiz de Austri, J. High Energy Phys. 12, 034 (2002); M. E. Gómez and J. D. Vergados, Phys. Lett. B 512, 252 (2001).

[8] A. Bottino, F. Donato, N. Fornengo, and S. Scopel, Phys. Rev. D 59, 095003 (1999); ibid. 59, 095004 (1999); ibid. 63, 125003 (2001); R. Arnowitt and P. Nath, ibid. 60, 044002 (1999); R. Arnowitt, B. Dutta, and Y. Santoso, Nucl. Phys. B606, 59 (2001).

[9] J. Ellis, J.S. Hagelin, D.V. Nanopoulos, K.A. Olive, and M. Srednicki, Nucl. Phys. B238, 453 (1984); see also H. Goldberg, Phys. Rev. Lett. 50, 1419 (1983).

[10] A. Melchiorri and J. Silk, Phys. Rev. D 66, 041301 (2002).

[11] C. L. Bennett et al., astro-ph/0302207; D. N. Spergel et al., astro-ph/0302209.

[12] S. L. Bridle, O. Lahav, J. P. Ostriker, and P. J. Steinhardt, astro-ph/0303180.

[13] M. Drees, M. M. Nojiri, D. P. Roy, and Y. Yamada, Phys. Rev. D 56, 276 (1997); ibid. 64, 039901(E) (2001); see also M. Drees, Y. G. Kim, M. M. Nojiri, D. Toya, K. Hasuko, and T. Kobayashi, ibid. 63, 035008 (2001).

[14] V. Berezinsky, A. Bottino, J. R. Ellis, N. Fornengo, G. Mignola, and S. Scopel, Astropart. Phys. 5, 1 (1996); P. Nath and R. Arnowitt, Phys. Rev. D 56, 2820 (1997); V. Bertin, E. Nezri, and J. Orloff, J. High Energy Phys. 02, 046 (2003).

[15] J. R. Ellis, T. Falk, G. Ganis, K. A. Olive, and M. Schmitt, Phys. Rev. D 58, 095002 (1998); J. R. Ellis, T. Falk, G. Ganis, and K. A. Olive, ibid. 62, 075010 (2000). 
[16] J. R. Ellis, K. A. Olive, and Y. Santoso, Phys. Lett. B 539, 107 (2002).

[17] J. R. Ellis, T. Falk, K. A. Olive, and Y. Santoso, Nucl. Phys. B652, 259 (2003).

[18] R. Arnowitt and P. Nath, Phys. Rev. D 46, 3981 (1992).

[19] V. D. Barger, M. S. Berger, and P. Ohmann, Phys. Rev. D 49, 4908 (1994).

[20] W. de Boer, R. Ehret, and D. I. Kazakov, Z. Phys. C 67, 647 (1995).

[21] M. Carena, J. R. Ellis, A. Pilaftsis, and C. E. Wagner, Nucl. Phys. B625, 345 (2002).

[22] L. E. Ibáñez, C. Lopez, and C. Munoz, Nucl. Phys. B256, 218 (1985).

[23] S. P. Martin and M. T. Vaughn, Phys. Rev. D 50, 2282 (1994).

[24] T. Falk, K. A. Olive, L. Roszkowski, and M. Srednicki, Phys. Lett. B 367, 183 (1996); A. Riotto and E. Roulet, ibid. 377, 60 (1996); A. Kusenko, P. Langacker, and G. Segre, Phys. Rev. D 54, 5824 (1996); T. Falk, K. A. Olive, L. Roszkowski, A. Singh, and M. Srednicki, Phys. Lett. B 396, 50 (1997).

[25] T. Falk, K. A. Olive, and M. Srednicki, Phys. Lett. B 339, 248 (1994).

[26] Joint LEP 2 Supersymmetry Working Group, Combined LEP Chargino Results, up to $208 \mathrm{GeV}$, http://lepsusy.web.cern.ch/ lepsusy/www/inos_moriond01/charginos_pub.html

[27] Joint LEP 2 Supersymmetry Working Group, Combined LEP Selectron/Smuon/Stau Results, 183-208 GeV, http:// lepsusy.web.cern.ch/lepsusy/www/sleptons_summer02/ slep_2002.html

[28] LEP Higgs Working Group for Higgs boson searches, OPAL Collaboration, ALEPH Collaboration, DELPHI Collaboration and L3 Collaboration, Search for the Standard Model Higgs Boson at LEP, ALEPH-2001-066, DELPHI-2001-113, CERNL3-NOTE-2699, OPAL-PN-479, LHWG-NOTE-2001-03, CERN-EP/2001-055, hep-ex/0107029; Searches for the neutral Higgs bosons of the MSSM: Preliminary combined results us- ing LEP data collected at energies up to $209 \mathrm{GeV}$, LHWGNOTE-2001-04, ALEPH-2001-057, DELPHI-2001-114, L3NOTE-2700, OPAL-TN-699, hep-ex/0107030; LHWG Note/ 2002-01, http://lephiggs.web.cern.ch/LEPHIGGS/papers/ July2002_SM/index.html

[29] Y. Okada, M. Yamaguchi, and T. Yanagida, Prog. Theor. Phys. 85, 1 (1991); J. R. Ellis, G. Ridolfi, and F. Zwirner, Phys. Lett. B 257, 83 (1991); H. E. Haber and R. Hempfling, Phys. Rev. Lett. 66, 1815 (1991).

[30] S. Heinemeyer, W. Hollik, and G. Weiglein, Comput. Phys. Commun. 124, 76 (2000); S. Heinemeyer, W. Hollik, and G. Weiglein, Eur. Phys. J. C 9, 343 (1999).

[31] CLEO Collaboration, M. S. Alam et al., Phys. Rev. Lett. 74, 2885 (1995) as updated in S. Ahmed et al., CLEO CONF 9910; BELLE Collaboration, BELLE-CONF-0003, contribution to the 30th International conference on High-Energy Physics, Osaka, 2000. See also Belle Collaboration, K. Abe et al., hep-ex/0107065; BaBar Collaboration, hep-ex/0110010; K. Chetyrkin, M. Misiak, and M. Munz, Phys. Lett. B 400, 206 (1997); ibid. 425, 414(E) (1997); T. Hurth, hep-ph/0106050; C. Degrassi, P. Gambino, and G. F. Giudice, J. High Energy Phys. 12, 009 (2000); M. Carena, D. Garcia, U. Nierste, and C. E. Wagner, Phys. Lett. B 499, 141 (2001); P. Gambino and M. Misiak, Nucl. Phys. B611, 338 (2001); D. A. Demir and K. A. Olive, Phys. Rev. D 65, 034007 (2002).

[32] Muon g-2 Collaboration, G. W. Bennett et al., Phys. Rev. Lett. 89, 101804 (2002); ibid. 89, 129903(E) (2002).

[33] M. Davier, S. Eidelman, A. Hocker, and Z. Zhang, Eur. Phys. J. C 27, 497 (2003); see also K. Hagiwara, A. D. Martin, D. Nomura, and T. Teubner, Phys. Lett. B 557, 69 (2003); F. Jegerlehner, unpublished, as reported in M. Krawczyk, Acta Phys. Pol. B 33, 2621 (2002).

[34] G. Jungman, M. Kamionkowski, and K. Griest, Phys. Rep. 267, 195 (1996); http://t8web.lanl.gov/people/jungman/neutpackage.html 